\title{
Pressions interstitielles dans les galeries en charge
}

\section{Pore pressure in pressure tunnels}

PAR

\author{
G. SAUVAGE DE SAINT-MARC, \\ DIRECTEUR GÉNÉRAL DE LA SOGRẺAH
}

M: BOUVARD ET MA MIN-YUAN

INGÉNIEURS A LA SOGRÉAH

\begin{abstract}
Dans une première partie, les auteurs étudient l'équilibre élastique d'une canalisation soumise $\dot{a}$ une pression intérieure, limitée par une paroi épaisse, voire infinie dans certains cas (tunnel par exemple).

Cette pression est supposée appliquée d'abord sur la paroi extérieure d'un tube épais, puis à une distance donnée de l'axe du cylindre. Enfin, la loi de pression en fonction du rayon est supposée correspondre $\dot{a}$ un écoulement poreux, la pression étant alors déterminée par la loi de Darcy.

Dans une seconde partie, ils étudient le cas de l'équilibre d'une galerie en charge creusée dans un massif rocheux, en admettant que le rocher se comporte comme un corps parfaitement poreux.

Les auteurs en déduisent quelques conséquences importantes concernant l'équilibre des revêtements des tunnels en charge, spécialement si la pression varie en fonction du temps : la pression ou la sous-pression encaissée par le revêtement dépend en grande partie de la perméabilité relative des matériaux en présence; il s'agit d'un problème d'hydraulique « souterraine ». Ceci conduit $\dot{a}$ penser que les calculs purement mécaniques des contraintes, suivant les hypothèses habituelles (pression supposée s'exercer da la surface intérieure de la cunalisation) n'ont guère de signification.
\end{abstract}

In the first part of this article the authors investigate the elastic equilibrium of an internally pressurized tube, the walls of which are thick and, in some cases, even infinite, as in the case of tunnels.

Pressure is first assumed to be applied to an external wall of a thick tube and then at a given distance from the axis of the cylinder. The radial pressure distribution is assumed to be the same as that for flow in a porous medium and the pressure can then be determined by Darey's law.

In the second part of the article, the authors investigate the equilibrium of a pressure tunnel driven through rock and assume that the rock behaves as a completely porous body.

Important deductions are made concerning the equilibrium of pressure tunnel linings, especially for the case where pressure varies with time. The pressures to which both sides of the lining are subjected are largely dependent upon the relative permeability of the materials under consideration. The problem is one of "underground" hydraulics. This leads to the conclusion that purely mechanical stress calculations, based on the usual assumption concerning the pressure to which the internal surface of the tube is subjected, have hardly an! significance.

On sait que les pressions exercées par l'eau à l'intérieur des ouvrages massifs sont susceptibles de jouer un rôle important, voire déterminant, dans leur équilibre; en particulier pour les barrages, l'analyse de leurs conditions de résistance a fait des progrès extrêmement importants du jour où, à la suite de Maurice Lévy, on a considéré l'effet des sous-pressions à l'intérieur du massif en béton. Celui-ci a admis que l'existence de la sous-pression était liée à la présence d'une fissure dans le béton. D'autres auteurs admettent, au contraire, que le matériau se comporterait comme un corps poreux, et qu'à tout écoulement interstitiel correspondrait l'apparition d'une " pression de courant » provoquant la sous-pression. On a discuté et on pourrait encore discuter sur le point de savoir laquelle de ces deux interprétations est la plus satisfaisante. 
De toutes façons, les pressions interstitielles nous paraissent jouer également un rôle fondamental dans les galeries en charge. Ces ouvrages sont utilisés de plus en plus dans les aménagements hydroélectriques, notamment pour tirer le parti maximum de l'utilisation d'une réserve artificielle.

Il nous a paru intéressant de reprendre les théories concernant l'équilibre de ces ouvrages en faisant jouer aux pressions interstitielles le rôle qu'à notre avis elles méritent. On suppose alors que la pression soit appliquée non plus aux parois, mais que les différents milieux (béton, rocher,...) se comportent comme des corps poreux où l'eau peut s'infiltrer. La sous-pression se répartit donc suivant une certaine loi, sur laquelle la théorie de Darcy apporte quelques éclaircissements.

Notre exposé comprendra deux parties :

$1^{\circ}$ Nous avons étudié dans cette partie les états élastiques du tuyau d'épaisseur finie (cas d'une conduite) ou infinie (cas du rocher creusé d'une ouverture circulaire). Les calculs des différents cas de charge sont faits avec la théorie de l'élasticité appliquée à un milieu homogène et poreux. Les formules obtenues sónt utiles pour le traitement du problème posé ici : la galerie en charge. Des remarques et des conséquences y sont indiquées pour faciliter les applications pratiques.

2' Nous développons quelques considérations concernant la distribution des pressions dans la galerie et l'influence de leurs variations. Nous en déduisons quelques conséquences pratiques concernant l'équilibre des galeries soumises à une charge qui, en fait, subit des variations constantes par suite du fonctionnement même des usines hydrauliques qu'elles alimentent.

Au moment de la rédaction de cette étude, nous avons eu connaissance de l'intéressant article de MM. Zienkiewicz et Parker, paru dans Water Power de janvier 1958, où le problème du tuyau épais est mis en équations à partir des mêmes idées directrices; les calculs ont été faits par des procédés différents, mais les résultats concernant les contraintes, qui seules sont données dans cet article, sont comparables à ceux que nous avons obtenus, après simplification et redressement de quelques erreurs typographiques.

\section{1 ${ }^{\text {re }}$ PARTIE. - ETUDE ELASTIQUE}

Pour mieux préciser les hypothèses des calculs, nous commençons par le rappel des équations générales à trois dimensions applicables aux milieux homogènes et poreux.

Il est connu que pour traiter les influences des pressions interstitielles sur l'état élastique d'un milieu poreux, on a besoin des coefficients de ce milieu :,

$$
\begin{aligned}
& m \text { : coefficient volumique de porosité hydraulique, } \\
& \eta \text { : coefficient superficiel de porosité hydraulique. }
\end{aligned}
$$

Une fois ces coefficients donnés, on peut établir les équations de la façon suivante :

En prenant par la pensée une coupe afin d'examiner l'état de contraintes däns ce milieu, on sait que si $\sigma, \tau$, sont les contraintes normale et tangentielle de cette coupe, les forces agissant sur la surface $\mathrm{S}$ de cette coupe sont définies par:

$$
\begin{aligned}
& \text { force normale }=\sigma \mathrm{S}-\eta_{\mathrm{SP}}=(\sigma-\eta \mathrm{P}) \mathrm{S} \\
& \text { force tangentielle }=\tau \mathrm{S}
\end{aligned}
$$

où $\mathrm{P}$ est la pression hydraulique qui dépend du point considèré.

Ainsi, en se rapportant aux axes triorthogonaux oxyz, avec oz orienté vers le sens inverse de la pesanteur, on a les équations d'équilibre suivantes dans le milieu solide homogène:

$$
\begin{aligned}
& \frac{\partial \sigma_{m}}{\partial x}+\frac{\partial \tau_{x y}}{\partial y}+\frac{d \tau_{x z}}{\partial z}=\frac{\partial(\eta \mathrm{P})}{\partial x} \\
& \frac{\partial \tau_{x y}}{\partial x}+\frac{\partial \sigma_{y}}{\partial y}+\frac{\partial \tau_{y z}}{\partial z}=\frac{\partial(\eta \mathrm{P})}{\partial y} \\
& \frac{\partial \tau_{x z}}{\partial x}+\frac{\partial \tau_{y z}}{\partial y}+\frac{\partial \sigma_{z}}{\partial z}-(\mathrm{M}+m) \rho g=\frac{\partial \eta \mathrm{P}}{\partial z}
\end{aligned}
$$


avec :

$$
\begin{aligned}
& \varrho=\text { la densité de l'eau, } \\
& g=\text { l'accélération de la pesanteur, } \\
& \mathrm{M}=\text { la densité du solide formant le milieu poreux. }
\end{aligned}
$$

En désignant alors par $u, v, w$ les déplacements du solide suivant les axes, on a, d'après la théorie de l'élasticité :

$$
\begin{array}{ll}
s_{x}=\frac{\partial u}{\partial x} & \gamma_{y y}=\frac{\partial v}{\partial x}+\frac{\partial u}{\partial y} \\
\varepsilon_{y}=\frac{\partial v}{\partial y} & \gamma_{y z}=\frac{\partial w}{\partial y}+\frac{\partial v}{\partial z} \\
\varepsilon_{z}=\frac{\partial w}{\partial z} & \gamma_{z x}=\frac{\partial u}{\partial z}+\frac{\partial w}{\partial x}
\end{array}
$$

(et les équations (4) sont valables pour les petites déformations et les petites rotations seulement).

$$
\begin{array}{ll}
\varepsilon_{x}=\frac{1}{\mathrm{E}}\left[\sigma_{x}-\nu\left(\sigma_{y}+\sigma_{z}\right)\right] & \gamma_{x y}=\frac{2(1+\nu)}{\mathrm{E}} \tau_{x y} \\
\varepsilon_{y / y}=\frac{1}{\mathrm{E}}\left[\sigma_{y}-\nu\left(\sigma_{z}+\sigma_{x}\right)\right] & \gamma_{y z}=\frac{2(1+\nu)}{\mathrm{E}} \tau_{y z z} \\
\varepsilon_{z}=\frac{1}{\mathrm{E}}\left[\sigma_{\tilde{z}}-\nu\left(\sigma_{x}+\sigma_{y}\right)\right] & \gamma_{z x}=\frac{2(1+\nu)}{\mathrm{E}} \tau_{z x}
\end{array}
$$

avec :

$$
\left.\begin{array}{c}
\text { E : coefficient de Young } \\
\vee: \text { coefficient de Poisson }
\end{array}\right\} \text { du solide considéré }
$$

(et les équations (5) sont valables dans le domaine élastique, c'est-à-dire des contraintes faibles).

En principe, on doit dans les recherches étudier le système formé par (3), (4), (5). Mais il est bien connu que, dans la pratique, on assimile, suivant le cas, les problèmes posés à des problèmes plans, soit de contraintes planes, soit de déformations planes, pour faciliter les calculs.

Pour les études de l'influence des pressions interstitielles dans les ouvrages mis au contact de l'eau, nous pensons, comme beaucoup d'autres, que les cas à considérer sont presque tous de déformations planes. C'est ainsi que nous allons étudier le problème des tuyaux épais dans le cas de déformations planes.

En apparence, le problème du tuyau épais est un problème de révolution. Or, pour que le problème soit vraiment de révolution, il faut -- si l'axe du tuyau est horizontal - que l'on supprime l'effet de la pesanteur, c'est-à-dire qu'on prenne $g=0$ dans (3).

Cette méthode serait rigoureuse si on cherchait à définir l'état de contrainte des tuyaux sous l'influence de la pression qui se surajoute à l'état de contrainte correspondant à l'équilibre sous l'influence de la pesanteur. Reste l'hypothèse concernant la pression, supposée implicitement, elle aussi, de révolution. Nous reviendrons sur ce point en précisant les valeurs de pressions prises en compte.

Considérons alors les axes cylindriques $o r, \theta, z$ tels que :

$$
\begin{aligned}
& o z \text { coïncide avec l'axe du tuyau, } \\
& \text { or coincide avec un rayon du tuyau, } \\
& \theta \text { exprime l'angle trigonométrique du rayon vecteur, }
\end{aligned}
$$

et désignons les déplacements par :

$$
\begin{aligned}
& u \text { dans la direction } o r \\
& v \text { dans la direction } \theta, \\
& w \text { dans la direction } o z \text {. }
\end{aligned}
$$

On sait que $v=0, w=0$, pour les problèmes de révolution et à déformation planes; les systèmes (3), (4), (5), se réduisent dans ce cas à : 
où :

$$
\left.\begin{array}{c}
\frac{d \sigma_{r}}{d r}+\frac{1}{r}\left(\sigma_{r}-\sigma_{t}\right)=\frac{d \eta \mathrm{P}(r)}{d r} \\
\sigma_{z}=\nu\left(\sigma_{r}+\sigma_{t}\right) \\
\varepsilon_{r}=\frac{d u}{d r}=\frac{1-v^{2}}{\mathrm{E}}\left[\sigma_{r}-\frac{v}{1-v} \sigma_{t}\right] \\
\varepsilon_{t}=\frac{u}{r}=\frac{1-v^{2}}{\mathrm{E}}\left[\sigma_{t}-\frac{v}{1-v} \sigma_{r}\right]
\end{array}\right\}
$$

$\sigma_{r}$ désigne la tension normale dans la direction or positive,

$\sigma_{t}$ désigne la tension normale dans la direction de $\theta$ positive.

Nous devons alors résoudre, pour la recherche de l'influence des pressions interstitielles dans les tuyaux épais, problème supposé de révolution, le système formé par (6), (7), (8). Or, il est bien connu que la résolution dudit système consiste à résoudre l'équation suivante :

$$
\frac{d^{2} u}{d r^{2}}+\frac{1}{r} \frac{d u}{d r}-\frac{u}{r^{2}}=\frac{(1+v)(1-2 v)}{\mathrm{E}(1-v)} \cdot \frac{d \eta \mathrm{P}(r)}{d r}
$$

obtenue en éliminant $\sigma_{r}, \sigma_{t}$ dans les équations (6), (7) et (8).

Il est à remarquer que dans les cas où : $\eta=0$ (c'est-à-dire qu'il n'y a pas d'eau infiltrée dans le milieu poreux), ou bien si :

$$
\frac{d \mathbf{P}(r)}{d r}=0
$$

(c'est-à-dire que l'eau infiltrée dans le milieu poreux est de pression constante), la solution de (9) donne les expressions suivantes qui sont bien connues :

$$
\left.\begin{array}{rl}
\frac{u}{r} & =\frac{\mathrm{A}}{r^{2}}+\mathrm{B} \\
\sigma_{r} & =\frac{\mathrm{E}}{(1+v)(1-2 v)}\left[-(1-2 v) \frac{\mathrm{A}}{r^{2}}+\mathrm{B}\right] \\
\sigma_{t} & =\frac{\mathrm{E}}{(1+v)(1-2 v)}\left[(1-2 v) \frac{\mathrm{A}}{r^{2}}+\mathrm{B}\right] \\
\sigma_{2} & =\frac{\mathrm{E}}{(1+v)(1-2 v)} \cdot 2 \vee \mathrm{B}
\end{array}\right\}
$$

oủ A, B sont deux constantes d'intégrations. Et nous allons utiliser (9) pour traiter les problèmes exposés ci-après.

I : Nous nous permettons de reproduire ici les calculs courants des tuyaux épais, tout en considérant les trois cas suivants :

A. Quel est l'état élastique du tuyau épais, de rayons $a, \mathrm{R}(a<R)$, étanche $\dot{a} r=a$, de pression hydraulique intérieure $p$, avec la possibilité de se déplacer lorsque $r=\mathrm{R}$.

Les conditions aux limites sont les suivantes:

$$
\begin{aligned}
& \sigma_{r=a}=-p \\
& \sigma_{r=\mathbb{R}}=0
\end{aligned}
$$

Un calcul très simple d'après (9)' nous redonne la solution bien connue :

$$
\begin{aligned}
\frac{r}{u} & =\frac{1+v}{\mathrm{E}} \frac{a^{2} p}{\mathrm{R}^{2}-a^{2}}\left[\frac{\mathrm{R}^{2}}{r^{2}}+(1-2 v)\right] \\
\sigma_{r} & =\frac{a^{2} p}{\mathrm{R}^{2}-a^{2}}\left[1-\frac{\mathrm{R}^{2}}{r^{2}}\right] \\
\sigma_{t} & =\frac{a^{2} p}{\mathrm{R}^{2}-a^{2}}\left[1+\frac{\mathrm{R}^{2}}{r^{2}}\right] \\
\sigma_{z} & =\frac{a^{2} p}{\mathrm{R}^{2}-a^{2}} 2
\end{aligned}
$$


avee :

$$
\frac{u_{a}}{a}=\frac{1+\nu}{\mathrm{E}} \cdot \frac{\mathrm{R}^{2}+(1-2 v) a^{2}}{\mathrm{R}^{2}-a^{2}} p
$$

Il est à remarquer, d'après $(10)_{a}$, qu'à $r=b(a<b<\mathrm{R})$, on a :

$$
\sigma_{b}=-\frac{\mathrm{R}^{2}-b^{2}}{\mathrm{R}^{2}-a^{2}} \cdot \frac{a^{2}}{b^{2}} \cdot p
$$

et on peut alors écrire pour les états élastiques définis par $(10)_{a}$, mais situés dans l’intervalle $b \leqslant r \leqslant \mathrm{R}$ :

$$
\begin{aligned}
\frac{u}{r} & =-\frac{1+\nu}{\mathrm{E}} \cdot \frac{b^{2} \sigma_{b}}{\mathrm{R}^{2}-b^{2}} \cdot\left[\frac{\mathrm{R}^{2}}{r^{2}}+(1-2 v)\right] \\
\sigma_{r} & =-\frac{b^{2}}{\mathrm{R}^{2}-b^{2}} \sigma_{b}\left[1-\frac{\mathrm{R}^{2}}{r^{2}}\right] \\
\sigma_{t} & =-\frac{b^{2}}{\mathrm{R}^{2}-b^{2}} \sigma_{b}\left[1+\frac{\mathrm{R}^{2}}{r^{2}}\right] \\
\sigma_{z} & =-\frac{b^{2} \sigma_{b}}{\mathrm{R}^{2}-b^{2}}-2 \vartheta
\end{aligned}
$$

D'après $(12)_{a},(10)_{a}$, il est très facile de voir que les contraintes $\sigma_{r}, \sigma_{t}$ diminuent en valeur absolue lorsque $r$ augmente. Et quand $\mathrm{R} \rightarrow \infty$, les $\sigma_{r}, \sigma_{t}, \sigma_{z}$ tendent tous vers zéro pour $r$ très grand.

B. Quel est l'état élastique du tuyau épais de rayon: $a, \mathrm{R}(a<\mathrm{R})$; étanche da $r=a$; de pression hydraulique intérieure $p$, sans la possibilité de se déplacer lorsque $r=\mathbf{R}$ ?

Les conditions aux limites sont dans ce cas :

$$
\begin{aligned}
& \sigma_{r=a}=-p \\
& u_{r=\mathrm{R}}=0
\end{aligned}
$$

Un calcul sans difficultés, d’après $(9)^{\prime}$, nous donne la solution également bien connue :

$$
\begin{aligned}
\frac{u}{r} & =\frac{(1+\nu)(1-2 \vartheta)}{\mathrm{E}} \frac{a^{2} p}{(1-2 \nu) \mathrm{R}^{2}+a^{2}}\left[\frac{\mathrm{R}^{2}}{r_{2}}-1\right] \\
\sigma_{r} & =-\frac{a^{2} p}{(1-2 \vartheta) \mathrm{R}^{2}+a^{2}}\left[(1-2 \nu) \frac{\mathrm{R}^{2}}{r_{2}}+1\right] \\
\sigma_{t} & =\frac{a^{2} p}{(1-2 \nu) \mathrm{R}^{2}+a^{2}}\left[(1-2 \nu) \frac{\mathrm{R}^{2}}{r_{2}}-1\right] \\
\sigma_{z} & =-\frac{a^{2} p}{(1-2 \nu) \mathrm{R}^{2}+a^{2}} 2 \nu
\end{aligned}
$$

avec :

$$
\frac{u_{a}}{a}=\frac{(1+v)(1-2 v)}{\mathrm{E}} \frac{\mathrm{R}^{2}-a^{2}}{(1-2 v) \mathrm{R}^{2}+a^{2}} p
$$

Il est à remarquer, d'après $(10)_{b}$, qu'à $r=b(a<b<\mathrm{R})$, on $\mathbf{a}$ :

$$
\sigma_{b}=-\frac{(1-2 \nu) \mathrm{R}^{2}+b^{2}}{(1-2 v) \mathrm{R}^{2}+a^{2}} \cdot \frac{a^{2}}{b^{2}} \cdot p
$$

et on peut alors écrire, pour les états élastiques définis par $(10)_{b}$, mais situés dans l'intervalle $b \leqslant r \leqslant \mathbf{R}:$ 


$$
\begin{aligned}
\frac{u}{r} & =-\frac{(1+v)(1-2 v)}{\mathrm{E}}-\frac{b^{2} \sigma_{b}}{(1-2 v) \mathrm{R}^{2}+b^{2}}\left[\frac{\mathrm{R}^{2}}{r^{2}}-1\right] \\
\sigma_{r} & \left.=\frac{b^{2} \sigma_{b}}{(1-2 v) \mathrm{R}^{2}+b^{2}} \mid(1-2 v) \frac{\mathrm{R}^{2}}{r_{2}}+1\right] \\
\sigma_{t} & =-\frac{b_{2} \sigma_{b}}{(1-2 \nu) \mathrm{R}^{2}+b^{2}}\left[(1-2 v) \frac{\mathrm{R}^{2}}{r^{2}}-1\right] \\
\sigma_{z} & =\frac{b^{2} \sigma_{b}}{(1-2 \nu) \mathrm{R}^{2}+b^{2}} 2 \nu
\end{aligned}
$$

D'après $(12)_{b}(10)_{b}$, il est très facile de voir que les contraintes $\sigma_{r}, \sigma_{t}$ diminuent en valeur absolue lorsque $r$ augmente. Et quand $\mathrm{R} \rightarrow \infty$, les $\sigma_{r}, \sigma_{t}, \sigma_{z}$ tendent tous vers zéro pour $r$ très grand.

C. Quel est l'état élastique du tuyau de rayon a, creusé dans un milieu infini, étanche à $r=a$, avec la pression intérieure $p$ ?

Les conditions aux limites sont dans ce cas :

$$
\begin{aligned}
& \sigma_{r=a}=-p \\
& \sigma_{r}=\sigma_{t}=0 \quad \text { pour } \quad r \rightarrow \infty .
\end{aligned}
$$

Un calcul sans difficultés nous donne, d'après $(9)^{\prime}$, la solution suivante :

$$
\begin{aligned}
\frac{u}{r} & =\frac{1+\nu}{\mathrm{E}} \cdot p \frac{a^{2}}{r^{2}} \\
\sigma_{r} & =-p \frac{a^{2}}{r_{2}} \\
\sigma_{l} & =p \frac{a^{2}}{r_{2}} \\
\sigma_{\tilde{r}} & =0
\end{aligned}
$$

avec :

$$
\frac{u_{a}}{a}=\frac{1+\nu}{\mathrm{E}} p
$$

Il est à remarquer, d'après $(10)_{c}$, qu'à $r=b(a<b)$, on a :

$$
\sigma_{b}=-p \frac{a^{2}}{b^{2}}
$$

et on peut alors écrire, pour les états élastiques définis par $(10)_{c}$, mais situés dans l'intervalle $b \leqslant r:$

$$
\begin{aligned}
\frac{u}{r} & =-\frac{1+\nu}{\mathrm{E}} \sigma_{b} \frac{b^{2}}{r^{2}} \\
\sigma_{r} & =\sigma_{b} \frac{b^{2}}{r^{2}} \\
\sigma_{t} & =-\sigma_{b} \frac{b^{2}}{r^{2}} \\
\sigma_{\tilde{*}} & =0
\end{aligned}
$$

Nous signalons ici que les formules $(10)_{e},(11)_{c},(12)_{c},(13)_{c}$ ne sont autres choses que les formules limites, pour $\mathbf{R} \rightarrow \infty$, de celles obtenues dans les cas $A$ et $B$. En effet, si l'on fait dans ces dernières tendre $\mathrm{R}$ vers l'infini et $a / \mathrm{R}, b / \mathrm{R}$ vers zéro, on retrouve directement les formules $(10)_{c},(11)_{c},(12)_{c},(13)_{c}$.

Or, d'après $(10)_{e}$, on sait que pour $r \geqslant 5 a$, les contraintes ne sont plus que de $4 \%$ (ou moins) des contraintes à $r=a$.

De même, pour les tuyaux épais à épaisseur finie $\mathrm{R}$, mais $\mathrm{R}>5 a$, on voit qu'à $r=5 a$, on a : 


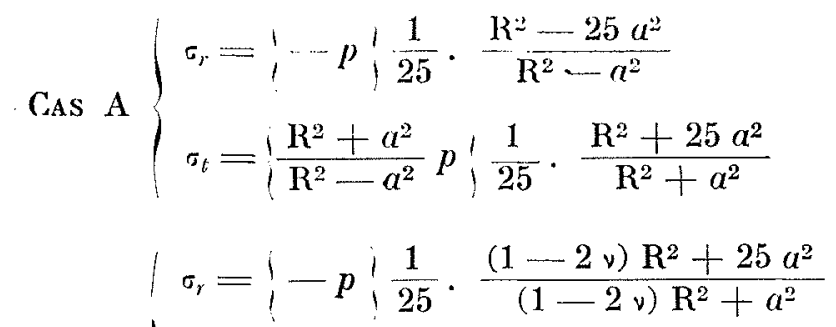

$$
\begin{aligned}
& \text { avec } \frac{\mathrm{R}^{2}-25 a^{2}}{\mathrm{R}^{2}-a^{2}}<1 \\
& \text { avec } \frac{\mathrm{R}^{2}+25 a^{2}}{\mathrm{R}^{2}+a^{2}}<\frac{50}{26} \\
& \text { avec } \frac{(1-2 v) \mathrm{R}^{2}+25 a^{2}}{(1-2 v) \mathrm{R}^{2}+a^{2}}<\frac{50(1-v)}{26-50 v}
\end{aligned}
$$

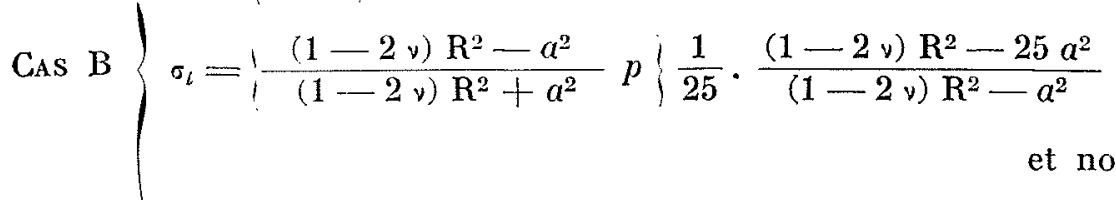

$$
\text { et normalement } \frac{(1-2 v) \mathrm{R}^{2}-25 a^{2}}{(1-2 v) \mathrm{R}^{2}-a^{2}}<1
$$

Pour le béton, on peut prendre $\nu=0,15$, ce qui montre que les contraintes à $r=5 a$ ne sont plus au maximum que de $9,2 \%$ (ou moins) des contraintes à $r=a$. Il est connu que, physiquement, on peut interpréter ce fait en disant que l'état élastique dans la zone de $r>5$ est négligeable; ou bien on dit que la zone d'influence des charges considérées se limite sensiblement à $r=5 a$.

Et d'après ces considérations, on peut parfaitement fixer la zone d'influence d'un tuyau épais en béton à 5-6 fois son rayon intérieur.

Ces résultats sont bien connus. Nous les avons indiqués ici en vue de les prendre dans la suite comme base de comparaison pour mieux saisir les phénomènes physiques dus à l'influence des eaux d'infiltration.

II. Nous admettons maintenant que la pression hydraulique n'est plus appliquẻe à la surface intérieure de la conduite, mais qu'elle pénètre la paroi même de la conduite tout en restant constante. C'est le cas du problème suivant:

«Quel est l'état élastique d'un tuyau épais de rayon intérieur a, avec la pression hydraulique p, cette pression s'étendant uniformément jusqu'd la couche $: r=b(b>a)$ (c'est-ádire que pour $r=b$, on a une couche imperméable)? 》

Les formules obtenues dans I ne sont plus valables pour ce problème. Mais on remarque qu'en partageant le tuyau en deux tranches:

$$
\begin{aligned}
& 1^{\text {re }} \text { où } a \leqslant r \leqslant b, \eta \neq 0 \quad \text { mais } \frac{d \mathrm{P}(r)}{d r}=0 \\
& 2^{\text {e }} \text { où } b \leqslant r \quad \eta=0
\end{aligned}
$$

la solution (9)' reste valable dans chacune de ces tranches. Pour faire les calculs complets, il faut tenir compte de la discontinuité dans la section $r=b$.

En effet, désignons par :

$$
\begin{aligned}
& \mid \begin{array}{l}
\square \\
- \\
- \\
-
\end{array} \\
& \begin{array}{l}
\sigma_{b-} \text { la tension radiale à } r=b-\varepsilon \\
\sigma_{b} \text { la tension radiale à } r=b+\varepsilon
\end{array}(\varepsilon \text { positif et } \rightarrow 0) \\
& r=b-\varepsilon \quad r=b \quad r=b+\varepsilon
\end{aligned}
$$

Nous avons alors :

$$
\sigma_{b-}=\sigma_{b}+\eta p
$$

Ainsi dans la tranche $a \leqslant r \leqslant b-\varepsilon$, on a les conditions aux limites :

$$
\begin{array}{ll}
r=a & \sigma_{r}=-(1-\eta) p \\
r=b-\varepsilon & \sigma_{r}=\sigma_{b}+\eta p
\end{array}
$$

ce qui donne, d'après $(9)^{\prime}$, la solution suivante : 


$$
\left.\begin{array}{l}
\left.\begin{array}{l}
u \\
r
\end{array}=\frac{1+v}{\mathrm{E}} \frac{1}{b^{2}-a^{2}}, b^{2} \sigma_{b} \mid \frac{a^{2}}{r^{2}}+(1-2 v)\right]+a^{2} p\left[\frac{b^{2}}{r^{2}}+(1-2 v)\right]+\eta p(1-2 \nu)\left(b^{2}-a^{2}\right) \\
\sigma_{r}=\frac{b^{2}}{b^{2}-a^{2}} \sigma_{b}\left(1-\frac{a^{2}}{r^{2}}\right)+\frac{a^{2}}{b^{2}-a^{2}} p\left(1-\frac{b^{2}}{r^{2}}\right)+\eta p \\
\sigma_{t}=\frac{b^{2}}{b^{2}-a^{2}} \sigma_{l}\left(1+\frac{a^{2}}{r^{2}}\right)+\frac{a^{2}}{b^{2}-a^{2}} p\left(1+\frac{b^{2}}{r^{2}}\right)+\eta p \\
\sigma_{z}=\frac{b^{2}}{b^{2}-a^{2}} \sigma_{b} \cdot 2 \nu+\frac{a^{2}}{b^{2}-a^{2}} \cdot p \cdot 2 \nu+2 v \eta
\end{array}\right\}
$$

et à $r=b-\varepsilon, \varepsilon \rightarrow 0$, on a :

$\left.\frac{u_{b}}{b}=\frac{1+\nu}{\mathrm{E}} \cdot \frac{1}{b^{2}-a^{2}} ; \sigma_{b}\left[a^{2}+(1-2 v) b^{2}\right]+p \cdot 2(1-\nu) a^{2}+\eta p(1-2 \nu)\left(b^{2}-a^{2}\right)\right\}$

Nous allons utiliser les formules (15) et (16) pour traiter le problème posé ici.

$A^{\circ}$. - Si le rayon extérieur du tuyau épais est $\mathrm{R}(b \leqslant \mathrm{R})$. Pour $r=\mathrm{R}$, le tuyau a la possibilité de se déplacer.

On sait, d'après $(13)_{a}$, qu'à $r=b+\varepsilon, \varepsilon \rightarrow 0$,

$$
\frac{u_{b}}{b}=-\frac{1+\nu}{\mathrm{E}} \frac{\mathrm{R}^{2}+(1-2 ; \nu) b^{2}}{\mathrm{R}^{2}-b^{2}} \sigma_{b}
$$

Ainsi, en égalant cette valeur de $u_{b}$ avec celle définie par (16), on trouve que :

$$
\sigma_{b}=-\frac{\mathrm{R}^{2}-b^{2}}{\mathrm{R}^{2}-a^{2}} \quad \frac{a^{2}}{b^{2}} p \cdot\left\{1+\frac{1-2 \nu}{2(1-v)} \eta \Phi\right\}
$$

avec :

$$
=\frac{b^{2}}{a^{2}}-1
$$

En tenant compte de $(17)_{a}$, on trouve, d'après (15), qu'entre $a \leqslant r \leqslant b-\varepsilon$ :

$$
\begin{aligned}
\frac{u}{r} & =\frac{1+\nu}{\mathrm{E}} \frac{a^{2} p}{\mathrm{R}^{2}-a^{2}} \frac{\left(\mathrm{R}^{2}\right.}{r^{2}}+(1-2 \nu)+\frac{1-2 \nu}{2(1-\nu)} \eta\left[\frac{\mathrm{R}^{2}+(1-2 \nu) b^{2}}{a^{2}}-2(1-\nu)-\frac{\mathrm{R}^{2}-b^{2}}{r^{2}}\right] \\
\sigma_{l} & =\frac{a^{2} p}{\mathrm{R}^{2}-a^{2}}\left(1-\frac{\mathrm{R}^{2}}{r^{2}}\right)+\eta p\left\{1-\frac{1-2 \nu}{2(1-\nu)} \frac{\mathrm{R}^{2}-b^{2}}{\mathrm{R}^{2}-a^{2}}\left(1-\frac{a^{2}}{r^{2}}\right)\right\} \\
\sigma_{t} & =\frac{a^{2} p}{\mathrm{R}^{2}-a^{2}}\left(1+\frac{\mathrm{R}^{2}}{r^{2}}\right)+\eta p\left\{1-\frac{1-2 \nu}{2(1-\nu)} \frac{\mathrm{R}^{2}-b^{2}}{\mathrm{R}^{2}-a^{2}}\left(1+\frac{a^{2}}{r^{2}}\right)\right\} \\
\sigma_{z} & =\frac{a^{2} p}{\mathrm{R}^{2}-a^{2}} \cdot 2 \nu+\eta p 2 \nu\left\{1-\frac{1-2 \nu}{2(1-\nu)} \frac{\mathrm{R}^{2}-b^{2}}{\mathrm{R}^{2}-a^{2}}\right\}
\end{aligned}
$$

avec :

$$
\frac{u_{a}}{a}=\frac{1+\nu}{\mathrm{E}} \quad \frac{\mathrm{R}^{2}+(1-2 \nu) a^{2}}{\mathrm{R}^{2}-a^{2}} p\left\{1+\eta(1-2 \nu) \frac{b^{2}-a^{2}}{\mathrm{R}^{2}+(1-2 \nu) a^{2}}\right\}
$$

Et en tenant compte de $(17)_{a}$, on trouve, d'après (13) $)_{a}$, qu'entre $b \leqslant r \leqslant \mathbf{R}$ :

$$
\begin{aligned}
& \frac{u}{r}=\frac{1+\nu}{\mathrm{E}} \frac{a^{2}}{\mathrm{R}^{2}-a^{2}} p\left\{1+\frac{1-2 \nu}{2(1-\nu)} \eta \cdot \Phi !\left[\frac{\mathrm{R}^{2}}{r^{2}}+(1-2 v)\right]\right. \\
& \sigma_{r}=\frac{a^{2}}{\mathrm{R}^{2}-a^{2}} p\left\{1+\frac{1-2 \nu}{2(1-\nu)} \eta \cdot \Phi !\left[1-\frac{\mathrm{R}^{2}}{r^{2}}\right]\right. \\
& \sigma_{t}=\frac{a^{2}}{\mathrm{R}^{2}-a^{2}} p\left\{1+\frac{1-2 \nu}{2(1-\nu)} \eta \cdot \Phi\right\}\left[1+\frac{\mathrm{R}^{2}}{r^{2}}\right] \\
& \sigma_{z}=\frac{a^{2}}{\mathrm{R}^{2}-a^{2}} p\left\{1+\frac{1-2 \nu}{2(1-\nu)} \eta \cdot \Phi\right. \\
&
\end{aligned}
$$


$B^{\circ}$. - Si le rayon extérieur du tuyau épais est $\mathrm{R}(b \leqslant \mathrm{R})$. Pour $r=\mathrm{R}$, le tuyau n'a pas de possibilités de se déplacer.

On sait, d'après $(13)_{c}$, qu'à $r=b+\varepsilon$, $\varepsilon$ positif mais $\rightarrow 0$ :

$$
\frac{u_{b}}{b}=-\frac{(1+v)(1-2 v)}{\mathrm{E}} \frac{\mathrm{R}^{2}-b^{2}}{(1-2 v) \mathbf{R}^{2}+b^{2}} \sigma_{b}
$$

Ainsi, en égalant cette valeur de $u_{b}$ avec celle définie par (16), on trouve que :

$$
\sigma_{b}=-\frac{(1-2 \nu) \mathrm{R}^{2}+b^{2}}{(1-2 \nu) \mathrm{R}^{2}+a^{2}} \frac{a^{2}}{\tilde{b}^{2}} p\left\{1+\frac{(1-2 \nu)}{2(1-\nu)} \eta \cdot \Phi\right\}
$$

où $\Phi$ est défini par (18) cité plus haut.

En tenant compte de $(17)_{b}$, on trouve, d'après (15), qu'entre $a \leqslant r \leqslant b-\varepsilon$ :

$$
\begin{aligned}
& \frac{u}{r}=\frac{(1+v)(1-2 v)}{\mathrm{E}} \frac{a^{2} p}{(1-2 v) \mathrm{R}^{2}+a^{2}}\left\{\frac{\mathrm{R}^{2}}{r^{2}}-1+\frac{\eta}{2(1-\nu)}\left[(1-2 \vartheta) \frac{\mathrm{R}^{2}-b^{2}}{a^{2}}\right.\right. \\
& \left.\left.+2(1-v)-\frac{(1--2 v) \mathrm{R}^{2}+b^{2}}{r^{2}}\right]\right\} \\
& \sigma_{r}=-\frac{-a^{2} p}{(1-2 \nu) \mathrm{R}^{2}+a^{2}}\left[1+\frac{(1-2 \nu) \mathrm{R}^{2}}{r^{2}}\right]+\eta p\left\{1-\frac{1-2 \nu}{2(1-\nu)} \frac{(1-2 \nu) \mathrm{R}^{2}+b^{2}}{(1-2 \nu) \mathrm{R}^{2}+a^{2}}\left(1-\frac{a^{2}}{r^{2}}\right)\right\} \\
& \sigma_{t}=\frac{a^{2} p}{(1-2 \nu) \mathrm{R}^{2}+a^{2}}\left[-1+\frac{(1-2 \nu) \mathrm{R}^{2}}{r^{2}}\right]+\eta p\left\{1-\frac{1-2 \nu}{2(1-\nu)} \frac{(1-2 \nu) \mathrm{R}^{2}+b^{2}}{(1-2 \nu) \mathrm{R}^{2}+a^{2}}\left(1+\frac{a^{2}}{r^{2}}\right)\right\} \\
& \left.\sigma_{z}=\frac{-a^{2} p}{(1-2 \nu) \mathrm{R}^{2}+a^{2}} 2 \nu+2 \nu \eta p \nmid 1-\frac{1-2 \nu}{2(1-\nu)} \cdot \frac{(1-2 \nu) \mathrm{R}^{2}+b^{2}}{(1-2 \nu) \mathrm{R}^{2}+a_{2}}\right\}
\end{aligned}
$$

avec :

$$
\frac{u_{a}}{a}=\frac{(1+\nu)(1-2 \nu)}{\mathrm{E}} \frac{\mathrm{R}^{2}-a^{2}}{(1-2 \nu) \mathrm{R}^{2}+a^{2}} p: 1-\eta \frac{b^{2}-a^{2}}{\mathrm{R}^{2}-a^{2}} !
$$

En tenant compte de $(17)_{b}$, on trouve, d'après (13) $)_{b}$, qu'entre $b \leqslant r \leqslant \mathbf{R}$ :

$$
\begin{aligned}
& \frac{u}{r}=\frac{(1+v)(1-2 \nu)}{\mathrm{E}} \cdot \frac{a^{2} p}{(1-2 v) \mathrm{R}^{2}+a^{2}}\left|1+\frac{1-2 \nu}{2(1-v)} \eta \cdot \Phi\right|\left\lceil\frac{\mathrm{R}^{2}}{r^{2}}-1\right\rceil \\
& \left.\sigma_{1}=-\frac{a^{2} p}{(1-2 v)} \frac{\mathrm{R}^{2}+a^{2}}{1} ! \frac{1-2 v}{2(1-\nu)} \eta^{1} !(1-2 v) \frac{\mathrm{R}^{2}}{r^{2}}+1\right\rceil \\
& \sigma_{t}=\frac{a^{2} p}{(1-2 \nu) R^{2}+a^{2}}\left\{1+\frac{1-2 \nu}{2(1-\nu)} \eta \Phi \nmid\left[(1-2 \nu) \frac{R^{2}}{r^{2}}-1\right]\right. \\
& \sigma_{\tilde{n}}=-\frac{a^{2} p}{(1-2 \nu) \mathrm{R}^{2}+u^{2}} 2 \nu\left\{1+\frac{1-2 \nu}{2(1-v)} \eta \Phi !\right.
\end{aligned}
$$

$C^{\lrcorner}$. - Si le tuyau est creusé dans un milieu infini,

On sait, d'après $(13)_{e}$, qu'à $r=b+\varepsilon$, s positif mais $\rightarrow 0$ :

$$
\frac{u_{b}}{b}=-\frac{1+v}{\mathrm{E}} \sigma_{b}
$$

Ainsi, en égalant cette valeur de $u_{b}$ avec celle définie par (16), on trouve que:

$$
\left.\sigma_{b}=-\frac{a^{2}}{b^{2}} p: 1+\frac{1-2 v}{2(1-y)} \eta \cdot d\right\}
$$

où $\Phi$ est toujours défini par (18) cité plus haut.

En tenant compte de $(17)_{c}$, on trouve, d'après (15), qu'entre $a \leqslant r \leqslant b-\varepsilon$ : 
avec :

$$
\begin{aligned}
\frac{u}{r} & =\frac{1+\nu}{\mathrm{E}} p\left\{\frac{a^{2}}{r^{2}}+\frac{1-2 \nu}{2(1-\nu)} \eta\left(1-\frac{a^{2}}{r^{2}}\right)\right\} \\
\sigma_{r} & =-p \frac{a^{2}}{r^{2}}+\eta p\left\{1-\frac{1-2 \nu}{2(1-\nu)}\left[1-\frac{a^{2}}{r^{2}}\right]\right\} \\
\sigma_{t} & =p \frac{a^{2}}{r^{2}}+\eta p\left\{1-\frac{1-2 \nu}{2(1-\nu)}\left[1+\frac{a^{2}}{r^{2}}\right]\right\} \\
\sigma_{z} & =\eta p \frac{\nu}{1-\nu}
\end{aligned}
$$

Et en tenant compte de $(17)_{c}$, on trouve, d'après $(13)_{c}$, que pour $b \leqslant r$ :

$$
\begin{aligned}
\frac{u}{r} & =\frac{1+\nu}{\mathrm{E}} p\left\{1+\frac{1-2 \nu}{2(1-\nu)} \eta \Phi\right\} \frac{a^{2}}{r^{2}} \\
\sigma_{r} & =-p\left\{1+\frac{1-2 \nu}{2(1-\nu)} \eta \Phi\right\} \frac{a^{2}}{r^{2}} \\
\sigma_{t} & =p\left\{1+\frac{1-2 \nu}{2(1-\nu)} \eta \Phi\right\} \frac{a^{2}}{r^{2}} \\
\sigma_{z} & =0
\end{aligned}
$$

Nous signalons ici aussi que les formules $(17)_{c},(19)_{e},(20)_{c},(21)_{c}$ ne sont autres choses que les formules limites, pour $\mathrm{R} \rightarrow \infty$, de celles obtenues dans les cas $A$ et B. En effet, si l'on fait, dans ces dernières, tendre $\mathrm{R}$ vers l'infini et $a / \mathrm{R}, b / \mathrm{R}$ vers zéro, on retrouve directement les formules $(17)_{c}$, (19) ${ }_{c}$, $(20)_{c},(21)_{c}$.

Or, on peut tirer en général de ces calculs les conclusions suivantes :

Un tuyau épais, chargé par la pression hydraulique p à son intérieur infiltrée d'une façon uniforme jusqu'à $r=b$, voit son état de tension modifié de façon importante par rapport au cas oì. la pression s'exercerait seulement à la surface, telle que :

$1^{\circ} \grave{a} r=a$ :

$\sigma_{r}$ est devenu $-p(1-\eta)$, c'est-à-dire diminue en valeur absolue;

$\sigma_{t}$ a augmenté dans les cas $\mathrm{A}$ et $\mathrm{C}$,

a augmenté (pour $b<\sqrt{\nu \mathrm{R}^{2}+(1-v) /(1-2 \nu) a^{2}}$ ),

ou diminué (pour $\left.b>\sqrt{v \mathrm{R}^{2}+(1-v) /(1-2 v) a^{2}}\right)$, dans le cas $\mathrm{B}$.

$2^{\circ}$ Dans l'intervalle $a \leqslant r \leqslant b$, on a les variations suivantes pour les trois cas $\mathrm{A}, \mathrm{B}, \mathrm{C}$ :

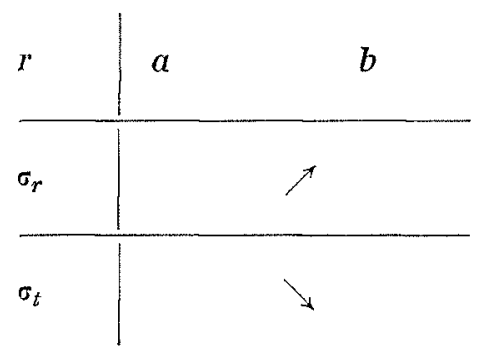

et les valeurs de $d \sigma_{r} / d r, d \sigma_{t} / d t$ sont plus modérées.

$3^{\circ}$ Dans l'intervalle $b \leqslant r$, on a les états élastiques du tuyau plus aggravés dans la proportion de 1 à :

$$
1+\eta \frac{1-2 \nu}{2(1-v)}\left(\frac{b^{2}}{a^{2}}-1\right)
$$


Et en conséquence, la zone d'influence définie dans le cas sans infiltration est étendue approximativement dans la proportion de $1 \grave{a}$ :

$$
\sqrt{1+n \frac{1-2 v}{2(1-v)}\left(\frac{b^{2}}{a^{2}}-1\right)}
$$

Il est à signaler que si $\mathrm{R}$ est grand par rapport à $a$, $b$, et que $b$ soit très grand par rapport à $a$, on a alors à la section $r=b$ (d'après (17) pour les cas $\mathrm{A}, \mathrm{B}, \mathrm{C}$ ) :

$$
\sigma_{b}=-p \frac{1-2 y}{2(1-v)} n
$$

qui ne dépend que de $v$ et $\eta$; à la section $r=\alpha$ :

$$
\frac{u_{a}}{a}=\frac{1+\nu}{\mathrm{E}} p
$$

III. Les calculs dans II sont faits en admettant que la pression interstitielle est constante jusqu'à une certaine valeur du rayon. Nous allons examiner le cas plus réel où la répartition de la pression interstitielle suit une loi normale, découlant de l'application de la loi de Darcy à l'écoulement de l'eau. Nous supposerons encore que l'ensemble du problème est de révolution. On peut objecter que ceci n'est pas tout à fait valable pour l'écoulement proprement dit. En effet, au lieu d'avoir affaire à un écoulement purement radial, on aurait à considérer un écoulement combiné, avec adjonction d'un ècoulement parallèle dû aux forces de gravité. Or, il est bien connu que dans le domaine des milieux poreux, où $d \mathrm{P} / d n$ est suffisamment grand, les autres effets sont en général négligeables (notamment celui de la pesanteur), et on peut y appliquer la loi de Darcy. Et c'est justement dans ce domaine que nous allons faire nos calculs.

Nous signalons en passant que nous examinerons plus longuement la validité de ces hypothèses de calcul dans la deuxième partie ci-après.

Si nous admettons que l'infiltration de l'eau dans le tuyau épais obéisse à la loi de Darcy, nous savons qu'au moment où l'infiltration de l'eau atteint $r=b$, la distribution de la pression hydraulique le long de $r$ est donnée par :

$$
\mathrm{P}(r)=p \cdot \frac{\log b / r}{\log b / a}, \quad a \leqslant r \leqslant b,
$$

c'est-à-dire :

$$
\frac{d \mathrm{P}(r)}{d r}=-\frac{p}{r \cdot \log b / a}
$$

L'équation (9) s'écrit alors pour $a \leqslant r \leqslant b$ :

$$
\frac{d^{2} u}{d r^{2}}+\frac{1}{r} \frac{d u}{d r}-\frac{u}{r^{2}}=-\frac{(1+\nu)(1-2 \nu)}{E(1-\nu)} \eta \frac{p}{r \log b / a}
$$

avec la solution générale suivante: ,

$$
\begin{aligned}
& \frac{u}{r}=\frac{\mathrm{A}}{r^{2}}+\mathrm{B}+1 / 2 \frac{(1+v)(1-2 \nu)}{\mathrm{E}(1-\nu)} \eta \frac{p}{\log b / a}[1 / 2-\log r] \\
& \left.\sigma_{r}=\frac{\mathrm{E}}{(1+\nu)(1-2 \nu)}\left[-(1-2 \nu) \frac{\mathrm{A}}{r^{2}}+\mathrm{B}\right]+\frac{\eta}{2(1-\nu)} p \frac{1}{\log b / a}\left[-\frac{1-2 \nu}{2}-\log r\right]\right\}_{(9)^{\prime \prime}} \\
& \sigma_{t}=\frac{\mathrm{E}}{(1+\nu)(1-2 v)}\left[(1-2 v) \frac{\mathrm{A}}{r^{2}}+\mathrm{B}\right]+\frac{\eta}{2(1-v)} p \frac{1}{\log b / a}\left[\frac{1-2 \nu}{2}-\log r\right] \\
& \sigma_{z}=\frac{2 \nu \mathrm{EB}}{(1+v)(1-2 v)}-\frac{\eta v p}{(1-v) \log b / a} \log r
\end{aligned}
$$

Désignons alors par $\sigma_{b}$ la tension normale à $r=b$; on sait que les constantes d'intégration $\mathrm{A}, \mathrm{B}$ de $(9)^{\prime \prime}$ sont définies par :

$$
\begin{array}{ll}
\sigma_{r}=-(1-\eta) p & \text { à } r=a \\
\sigma_{r}=\sigma_{b} & \text { à } r=b
\end{array}
$$


et un calcul simple montre que l'on a :

$$
\begin{aligned}
& \frac{u}{r}=\frac{1+\nu}{\mathrm{E}} \frac{b^{2} \sigma_{b}}{b^{2}-a^{2}}\left[\frac{a^{2}}{r^{2}}+(1-2 \nu)\right]+\frac{1+\nu}{\mathrm{E}} \frac{a^{2} p}{b^{2}-a^{2}}\left[\frac{b^{2}}{r^{2}}+(1-2 \nu)\right] \\
& +\frac{(1+v)(1-2 v)}{2 \mathrm{E}(1-v)} \eta p\left[-\frac{a^{2}}{b^{2}-a^{2}}\left\{\frac{b^{2}}{r^{2}}+(1-2 v)\right\}+\frac{(1-v)+\log b / r}{\log b / a}\right] \\
& \sigma_{r}=\frac{b^{2} \sigma_{b}}{b^{2}-a^{2}}\left(1-\frac{a^{2}}{r^{2}}\right)+\frac{a^{2} p}{b^{2}-a^{2}}\left(1-\frac{b^{2}}{r^{2}}\right) \\
& -\frac{\eta p}{2(1-\nu)}\left[(1-2 \nu) \frac{a^{2}}{b^{2}-a^{2}}\left(1-\frac{b^{2}}{r^{2}}\right)-\frac{\log b / r}{\log b / a}\right] \\
& \sigma_{\imath}=\frac{b^{2} \sigma_{b}}{b^{2}-a^{2}}\left(1+\frac{a^{2}}{r^{2}}\right)+\frac{a^{2} p}{b^{2}-a^{2}}\left(1+\frac{b^{2}}{r^{2}}\right) \\
& -\frac{\eta p}{2(1-v)}\left[(1-2 \nu) \frac{a^{2}}{b^{2}-a^{2}}\left(1+\frac{b^{2}}{r^{2}}\right)-\frac{1-2 \nu}{\log b / a}-\frac{\log b / r}{\log b / a}\right] \\
& \sigma_{z}=\frac{2 \vee b^{2} \sigma_{b}}{b^{2}-a^{2}}+\frac{2 \vee a^{2} p}{b^{2}-a^{2}}-\frac{\eta v p}{2(1-\nu)}\left[(1-2 v) \frac{2 a^{2}}{b^{2}-a^{2}}-\frac{1-2 \vee}{\log b / a}-\frac{\log b / r}{\log b / a}\right]
\end{aligned}
$$

et :

$$
\left.\begin{array}{rl}
\frac{u_{b}}{b}=\frac{1+\nu}{\mathrm{E}} \frac{(1-2 v) b^{2}+a^{2}}{b^{2}-a^{2}} & \sigma_{b}+\frac{2(1+v)(1-v)}{\mathrm{E}} \frac{a_{2}}{b^{2}-a^{2}} p \\
& +\frac{(1+v)(1-2 v)}{2 \mathrm{E}} \frac{\eta a^{2}}{b^{2}-a^{2}} p\left[\left(\frac{b^{2}}{a^{2}}-1\right) \frac{1}{\log b / a}-2\right]
\end{array}\right\}
$$

Nous allons utiliser les formules (22), (23) pour traiter les problèmes suivants :

Quel est l'état élastique d'un tuyau épais de rayon intérieur a, avec la pression hydraulique p, en admettant que cette pression s'infiltre en obéissant à la loi de Darcy jusqu'à $r=b$ ?

Nous allons considérer les trois cas suivants, comme dans les calculs précédents :

A. Le rayon extérieur du tuyau épais est $\mathrm{R}(b<\mathrm{R})$ et, à $r=\mathbf{R}$, le tuyau a la possibilité de se déplacer. Or, on sait, d'après $(13)_{a}$, qu'à $r=b$, la couche $r>b$ admet :

$$
\frac{u_{b}}{b}=-\frac{1+\nu}{\mathrm{E}} \frac{\mathrm{R}^{2}+(1-2 \nu) b^{2}}{\mathrm{R}^{2}-b^{2}} \sigma_{b}
$$

En égalant cette valeur de $u_{b}$ avec celle définie par (23), on trouve que :

$$
\sigma_{b}=-\frac{\mathrm{R}^{2}-b^{2}}{\mathrm{R}^{2}-a^{2}} \cdot \frac{a^{2}}{b^{2}} p\left\{1+\frac{1-2 v}{2(1-v)} \eta \cdot \varphi\right\}
$$

avec :

$$
\varphi=\left(\frac{b^{2}}{a^{2}}-1\right) \cdot \frac{1}{\log (b / a)^{2}}-1
$$

Ainsi, en tenant compte de $(24)_{a}$, on voit d'après (22), qu'entre $a<r<b$,

$$
\begin{aligned}
& \frac{u}{r}=\frac{1+\nu}{\mathrm{E}} \frac{a^{2}}{\mathrm{R}^{2}-a^{2}} p\left[\frac{\mathrm{R}^{2}}{r^{2}}+(1-2 \nu)\right]+\frac{(1+\nu)(1-2 \nu)}{2 \mathrm{E}(1-\nu)} \eta p \times \\
& \left\{-\frac{\mathrm{R}^{2}-b^{2}}{\mathrm{R}^{2}-a^{2}} \frac{\left(a^{2} / r^{2}\right)+(1-2 v)}{\log (b / a)^{2}}-\frac{a^{2}}{\mathrm{R}^{2}-a^{2}}\left[\frac{\mathrm{R}^{2}}{r^{2}}+(1-2 v)\right]+\frac{1-v+\log b / r}{\log b / a}\right\} \\
& \sigma_{r}=\frac{a^{2}}{\mathrm{R}^{2}-a^{2}} p\left(1-\frac{\mathrm{R}^{2}}{r^{2}}\right)-\frac{\eta p}{2(1-\nu)} \times \\
& \left.\left\{(1-2, y)\left[\frac{\mathrm{R}^{2}-b^{2}}{\mathrm{R}^{2}-a^{2}} \frac{1-\left(a^{2} / r^{2}\right)}{\log (b / a)^{2}}+\frac{a^{2}}{\mathrm{R}^{2}-a^{2}}\left(1-\frac{\mathrm{R}^{2}}{r^{2}}\right)\right]-\frac{\log b / r}{\log b / a}\right\}\right\}_{(26)_{a}} \\
& \sigma_{t}=\frac{a^{2}}{\mathrm{R}^{2}-a^{2}} p\left(1+\frac{\mathrm{R}^{2}}{r^{2}}\right)-\frac{\eta p}{2(1-v)} \times \text {. } \\
& \left\{(1-2 v)\left[\frac{\mathrm{R}^{2}-b^{2}}{\mathrm{R}^{2}-a^{2}} \frac{1+\left(a^{2} / r^{2}\right)}{\log (b / a)^{2}}+\frac{a^{2}}{\mathrm{R}^{2}-a^{2}}\left(1+\frac{\mathrm{R}^{2}}{r^{2}}\right)-\frac{1}{\log b / a}\right]-\frac{\log b / r}{\log b / a}\right\} \\
& \sigma_{z}=\nu\left[\frac{2 a^{2}}{\mathrm{R}^{2}-a^{2}} p-\frac{\eta p}{2(1-v)}\left\{(1-2 v)\left[-\frac{b^{2}-a^{2}}{\mathrm{R}^{2}-a^{2}} \frac{1}{\log b / a}+\frac{2 a^{2}}{\mathrm{R}^{2}-a^{2}}\right]-2 \frac{\log b / r}{\log b / a}\right\}\right)
\end{aligned}
$$


avec :

$$
\left.\frac{u_{a}}{a}=\frac{1+\nu}{\mathrm{E}} \cdot \frac{\mathrm{R}^{2}+(1-2 v) a^{2}}{\mathrm{R}^{2}-a^{2}} p \nmid 1+\frac{a^{2}}{\mathrm{R}^{2}+(1-2 v) a^{2}} \eta(1-2 v) \varphi\right\}
$$

Et en tenant compte de $(24)_{a}$, on trouve, d'après $(13)_{a}$, qu'entre $b \leqslant r \leqslant \mathrm{R}$ :

$$
\begin{aligned}
\frac{u}{r} & =\frac{1+\nu}{\mathrm{E}} \cdot \frac{a^{2}}{\mathrm{R}^{2}-a^{2}} p\left\{1+\frac{1-2 \nu}{2(1-\nu)} \eta \varphi\right\}\left[\frac{\mathrm{R}^{2}}{r^{2}}+(1-2 \nu)\right] \\
\sigma_{r} & =\frac{a^{2}}{\mathrm{R}^{2}-a^{2}} p\left\{1+\frac{1-2 \nu}{2(1-\nu)} \eta \varphi\right\}\left[1-\frac{\mathrm{R}^{2}}{r^{2}}\right] \\
\sigma_{i} & =\frac{a^{2}}{\mathrm{R}^{2}-a^{2}} p\left\{1+\frac{1-2 \nu}{2(1-\nu)} \eta \varphi\right\}\left[1+\frac{\mathrm{R}^{2}}{r^{2}}\right] \\
\sigma_{\tilde{*}} & =\frac{2 \nu a^{2}}{\mathrm{R}^{2}-a^{2}} p\left\{1+\frac{1-2 \nu}{2(1-\nu)} \eta \varphi\right\}
\end{aligned}
$$

B. Le rayon extérieur du tuyau épais est $\mathrm{R}(b<\mathrm{R})$ et, à $r=\mathrm{R}$, le tuyau n'a pas la possibilité de se déplacer.

Or, on sait, d'après $(13)_{b}$, qu'à $r=b$, la couche $r>b$ admet :

$$
\frac{u_{b}}{b}=-\frac{(1+\nu)(1-2 \nu)}{\mathrm{E}} \frac{\mathrm{R}^{2}-b^{2}}{(1-2 \nu) \mathrm{R}^{2}+b^{2}} \sigma_{\nu}
$$

En égalant cette valeur de $u_{b}$ avec celle définie par (23), on trouve que :

$$
\sigma_{b}=-\frac{(1-2 v) \mathbf{R}^{2}+b^{2}}{(1-2 v) \mathbf{R}^{2}+a^{2}} \frac{a^{2}}{b^{2}} p\left\{1+\frac{1-2 v}{2(1-v)} \gamma_{\varphi}\right\}
$$

où $\varphi$ est défini par (25) cité plus haut,

Ainsi, en tenant compte de $(24)_{b}$, on $a$, d'après (22), qu'entre $a<r<b$ :

$$
\begin{aligned}
& \frac{u}{r}=\frac{(1+v)(1-2 v)}{\mathrm{E}} \frac{a^{2} p}{(1-2 v) \mathrm{R}^{2}+a^{2}}\left[\frac{\mathrm{R}^{2}}{r^{2}}-1\right]+\frac{(1+v)(1-2 v)}{2 \mathrm{E}(1-v)} \eta p \\
& \left\{-\frac{(1-2 v) a^{2}}{(1-2 v) \mathrm{R}^{2}+a^{2}}\left[\frac{\mathrm{R}^{2}}{r^{2}}-1\right]+\frac{(1-v)+\log b / r}{\log b / a}-\frac{(1-2 v) \mathrm{R}^{2}+b^{2}}{(1-2 v)} \cdot \frac{\left(a^{2} / r^{2}\right)+(1-2 v)}{\log (b / a)^{2}}\right\} \\
& \sigma_{r}=-\frac{a^{2} p}{(1-2 \nu) \mathrm{R}^{2}+a^{2}}\left[1+(1-2 \nu) \frac{\mathrm{R}^{2}}{r^{2}}\right]-\frac{\eta p}{2(1-\nu)}\{(1-2 \nu) \times \\
& \left.\left[-\frac{a^{2}}{(1-2 v) \mathrm{R}^{2}+a^{2}}\left(1+(1-2 \nu) \frac{\mathrm{R}^{2}}{r^{2}}\right)+\frac{(1-2 \nu) \mathrm{R}^{2}+b^{2}}{(1-2 \nu) \mathrm{R}^{2}+a^{2}} \cdot \frac{1-\left(a^{2} / r^{2}\right)}{\log (b / a)^{2}}\right]-\frac{\log b / r}{\log b / a}\right\} \\
& \left.\sigma_{t}=\frac{a^{2} p}{(1-2 \nu) \mathrm{R}^{2}+a^{2}}\left[-1+(1-2 v) \frac{\mathrm{R}^{2}}{r^{2}}\right]-\frac{r_{p} p}{2(1-v)}\right\}(1-2 \nu) \times \\
& \left\lceil\frac{a^{2}}{(1-2 v) \mathrm{R}^{2}+a^{2}}\left(-1+(1-2 v) \frac{\mathrm{R}^{2}}{r^{2}}\right)+\frac{(1-2 v) \mathrm{R}^{2}+b^{2}}{(1-2 v) \mathrm{R}^{2}+a^{2}} \frac{1+\left(a^{2} / r^{2}\right)}{\log (b / a)^{2}}-\frac{1}{\mathrm{Log} b / a}\right] \\
& \left.-\frac{\log b / r}{\log b / a}\right\} \\
& \sigma_{z}=-\frac{2 v a^{2} p}{(1-2 v) \mathbf{R}^{2}+a^{2}}-\frac{v \eta p}{2(1-v)}\{(1-2 v) \times \\
& \left.\left[-\frac{2 a^{2}}{(1-2 v) \mathrm{R}^{2}+a^{2}}+\frac{b^{2}-a^{2}}{(1-2 v) \mathrm{R}^{2}+a^{2}} \frac{1}{\log b / a}\right]-2 \frac{\log b / r}{\log b / a}\right\}
\end{aligned}
$$

avec :

$$
\frac{u_{a}}{a}=\frac{(1+v)(1-2 v)}{\mathrm{E}} \frac{\mathrm{R}^{2}-a^{2}}{(1-2 v) \mathrm{R}^{2}+a^{2}} p\left\{1-\eta \frac{a^{2}}{\mathrm{R}^{2}-a^{2}} \cdot \varphi\right\}
$$


El en tenant compte de $(24)_{b}$, on trouve, d'après $(13)_{b}$, qu'entre $b \leqslant r<\mathbf{R}$ :

$$
\begin{aligned}
\frac{u}{r} & =\frac{(1+\nu)(1-2 \nu)}{\mathrm{E}} \frac{a^{2} p}{(1-2 \nu) \mathrm{R}^{2}+a^{2}}\left\{1+\frac{1-2 \nu}{2(1-\nu)} \eta \varphi\right\}\left[\frac{\mathrm{R}^{2}}{r^{2}}-1\right] \\
\sigma_{r} & =-\frac{a^{2}}{(1-2 \nu) \mathrm{R}^{2}+a^{2}} p\left\{1+\frac{1-2 \nu}{2(1-\nu)} \eta \varphi\right\}\left[(1-2 \nu) \frac{\mathrm{R}^{2}}{r^{2}}+1\right] \\
\sigma_{t} & =\frac{a^{2}}{(1-2 \nu) \mathrm{R}^{2}+a^{2}} p\left\{1+\frac{1-2 \nu}{2(1-\nu)} \eta \varphi\right\}\left[(1-2 \nu) \frac{\mathrm{R}^{2}}{r^{2}}-1\right] \\
\sigma_{z} & =-\frac{2 \nu a^{2}}{(1-2 \nu) \mathrm{R}^{2}+a^{2}} p\left\{1+\frac{1-2 \nu}{2(1-\nu)} \eta \varphi\right\}
\end{aligned}
$$

C. Le tuyau est creusé dans un milieu infini.

On sait, d'après $(13)_{c}$, qu'à $r=b$, la couche $b<r$ admet :

$$
\frac{u_{b}}{b}=-\frac{1+v}{\mathrm{E}} \cdot \sigma_{b}
$$

En égalant cette valeur de $u_{b}$ avec celle définie par (23), on trouve que :

$$
\sigma_{b}=-\frac{a^{2}}{b^{2}} p\left\{1+\frac{1-2 \nu}{2(1-v)} \eta \varphi\right\}
$$

où $\varphi$ est toujours défini par (25) cité plus haut.

Ainsi, en tenant compte de $(24)_{c}$, on voit, d'après (22), qu'entre $a \leqslant r \leqslant b$ :

$$
\begin{aligned}
\frac{u}{r} & =\frac{1+\nu}{\mathrm{E}} p \frac{a^{2}}{r^{2}}+\frac{(1+\nu)(1-2 \nu)}{2 \mathrm{E}(1-\nu)} \eta p\left[-\frac{a^{2}}{r^{2}}+\frac{1-\left(a^{2} / r^{2}\right)}{\log (b / a)^{2}}+\frac{\log b / r}{\log b / a}\right] \\
\sigma_{r} & =-p \frac{a^{2}}{r^{2}}+\frac{\eta p}{2(1-\nu)}\left\{(1-2 v)\left[\frac{a^{2}}{r^{2}}-\frac{1-\left(a^{2} / r^{2}\right)}{\log (b / a)^{2}}\right]+\frac{\log b / r}{\log b / a}\right\} \\
\sigma_{t} & =p \frac{a^{2}}{r^{2}}+\frac{\eta p}{2(1-\nu)}\left\{(1-2 \nu)\left[-\frac{a^{2}}{r^{2}}+\frac{1-\left(a^{2} / r^{2}\right)}{\log (b / a)^{2}}\right]+\frac{\log b / r}{\log b / a}\right\} \\
\sigma_{z} & =\frac{\nu \eta p}{(1-\nu)} \quad \frac{\log b / r}{\log b / a}
\end{aligned}
$$

avec :

$$
\frac{u_{a}}{a}=\frac{1+\nu}{\mathrm{E}} \cdot p
$$

Et en tenant compte de $(24)_{c}$, on troure, d'après 13$)_{c}$, que pour $b<r$,

$$
\begin{aligned}
& \frac{u}{r}=\frac{1+\nu}{\mathrm{E}} p\left\{1+\frac{1-2 \nu}{2(1-\nu)} \eta \varphi\right\} \frac{a^{2}}{r^{2}} \\
& \sigma_{r}=-p\left\{1+\frac{1-2 \nu}{2(1-\nu)} \eta \varphi\right\} \frac{a^{2}}{r^{2}} \\
& \sigma_{t}=p\left\{1+\frac{1-2 \nu}{2(1-\nu)} \eta \varphi\right\} \frac{a^{2}}{r^{2}} \\
& \sigma_{z}=0
\end{aligned}
$$

Nous signalons aussi que les formules $(24)_{c},(26)_{c},(27)_{c},(28)_{c}$ ne sont autres choses que les formules limites, pour $\mathbf{R} \rightarrow \infty$, de celles obtenues dans les cas A et B. En effet, si l'on fait, dans ces dernières, tendre $\mathrm{R}$ vers l'infini et $a / \mathrm{R}, b / \mathrm{R}$ vers zéro, on retrouve directement les formules $(24)_{c}$, $(26)_{c},(27)_{c},(28)_{c}$.

L'application des calculs précédents conduirait aux résultats caractéristiques suivants : 
$1^{\circ}$ A $r=a$, on a toujours :

$$
\sigma_{r=a}=-p(1-\eta)
$$

$2^{\circ}$ Dans l'intervalle $b \leqslant r$, on a les contraintes dans les tuyaux augmentées dans la proportion de 1 à :

$$
1+\eta \cdot \frac{1-2 v}{2(1-v)}\left[\left(\frac{b^{2}}{a^{2}}-1\right) \frac{1}{\log (b / a)^{2}}-1\right]
$$

En conséquence, la zone d'influence dans le cas sans infiltration est majorée approximativement dans la proportion de 1 à :

$$
\sqrt{1+\eta \cdot \frac{1-2 v}{2(1-\nu)}\left[\left(\frac{b^{2}}{a^{2}}-1\right) \frac{1-}{\log (b / a)^{2}}-1\right]}
$$

Nous signalons en terminant que pour les mêmes valeurs de $a, b, \mathbf{R}$ :

c'est-à-dire :

$$
\frac{b^{2}}{a^{2}}-1>\left(\frac{b^{2}}{a^{2}}-1\right) \frac{1}{\log (b / a)^{2}}-1
$$

Dans le cas oủ $\mathrm{R}$ est très grand par rapport à $a$, $b$, et $b$ très grand par rapport à $a$, on, a d'après (24), pour les trois cas A, B, C :

et :

$$
\sigma_{b} \cong p \frac{1-2 v}{4(1-v)} \eta \cdot \frac{-1}{\log b / a}
$$

$$
\frac{u_{a}}{a}=\frac{1+v}{\mathbf{E}} p
$$

Et il est à remarquer qu'en comparant ces dernières valeurs avec celles obtenues dans le cas II, on a :

et :

$$
\mathrm{U}_{a} / a \text { des cas III }=\mathrm{U}_{a} / a \text { des cas II }
$$

$$
\sigma_{b} \text { des cas III }=\frac{1}{2 \log b / a} \cdot \sigma_{b} \text { des cas II. }
$$

Il nous reste dans cette partie à examiner les constantes à utiliser dans les applications pratiques. En effet, même pour les bétons, les valeurs à prendre dans les constantes élastiques : E, v, ne soulèvent pas de grandes difficultés, mais en revanche la valeur du coefficient superficiel de porosité $n$ à utiliser est un sujet qui a soulevé beaucoup de discussions d'ordre théorique et pratique. Il n'est pas notre intention d'entrer en détail dans ces discussions. Comme il est bien connu que :

$1^{\circ}$ Les bétons ont généralement le coefficient de porosité volumétrique :

$$
m=0,12-0,15
$$

et les ciments de ces bétons ont, de leur côté :

$$
m=0,27-0,54
$$

$2^{\circ}$ Des essais dans les laboratoires ont donné, pour la surface maximum à prendre en compte en ce qui concerne l'action de sous-pressions :

$$
\eta=0,85-1,0
$$

Nous remarquons alors qu'en homogénéisant un milieu de $m=0,54$, on peut trouver géométriquement que, pour ce milieu considéré :

$$
\eta=m^{2 / 3}=(0,54)^{2 / 3} \cong 2 / 3
$$

et que l'intervalle $(2 / 3)-1$ comprend bien à son intérieur les valeurs de $\eta$ obtenues par expérience.

Ceci peut être regardé comme une anomalie, explicable d'après nous par le fait que, à porosité 
volumétrique égale, le mode d'action des sous-pressions dépendrait localement du degré de fissurations microscopiques; ainsi, il nous parait très intéressant de prendre successivement:

$$
\begin{array}{ll}
\mathrm{A}^{\circ} & \eta=2 / 3 \\
\mathrm{~B}^{\circ} & \eta=1
\end{array}
$$

et de faire les deux calculs. Les résultats ainsi obtenus pourraient être considérés comme les deux bornes du phénomène physique. Et cette souplesse de calcul aide mieux à approcher la vérité.

\section{$2^{e}$ PARTIE. - CONSIDERATIONS PRATIQUES}

Nous avons indiqué les formules qui déterminent l'état élastique de la galerie dans le cadre des hypothèses de base : pression connue à l'intérieur du terrain. Pour utiliser judicieusement ces formules, il faut savoir comment va s'appliquer réellement la pression à l'intérieur du terrain. Nous allons exposer ci-après nos considérations :

Examinons d'abord le cas des galeries non revêtues.

Supposons que l'on puisse successivement remplir d'eau une galerie cylindrique creusée dans un rocher homogène et poreux; puis on la met en pression brusquement. Au début, la pression sera bien appliquée à la surface. Le débit de fuite peut être évalué par la théorie. Soit, comme précédemment, $b$ le rayon atteint par l'eau à l'intérieur du terrain, $a$ le rayon intérieur de la galerie, et $q$ le débit de fuite par unité d'angle et par unité de longueur; l'application de la loi de Darcy donne la relation suivante :

$$
\mathbf{H}=\mathbf{H}_{0}-\frac{q}{k} \log \frac{r}{a}
$$

avec : $\quad \mathbf{H}_{0}=\frac{p}{\sigma}, p$ pression appliquée à $r=a, \mathrm{H}=\mathrm{P}(r) / \bar{\omega}, \mathrm{P}(r)$ pression courante à la distance $r$ du centre de la galerie

et :

$$
q=\frac{k \mathrm{H}_{0}}{\log (b / a)}
$$

On voit donc que le débit de fuite serait théoriquement infini, initialement.

Mais ce débit de fuite va lui-même concourir à remplir une couche cylindrique de terrain, suivant un processus qu'on peut schématiser de la façon suivante:

Si $m$ est le coefficient de porosité volumétrique, le débit $q$ coulant pendant un temps $d t$ permeltra de remplix un volume de rocher égal à $m b$. db. La loi qui donne la variation du rayon de $b$ en fonction du temps serait donc la suivante:

$$
\begin{aligned}
& m b \cdot d b=q d t, \text { soit : } \\
& m b . d b=\frac{k \mathbf{H}_{0}}{\log (b / a)} d t
\end{aligned}
$$

Cetle équation s'intègre facilement et on aboutit à l'expression suivante :

$$
k \frac{H_{0}}{m} t=\frac{r^{2}}{2} \log \frac{r}{a}+\frac{a^{2}-r^{2}}{4}=\frac{r^{2}}{2}\left|\log \frac{r^{2}}{a}-\frac{1}{2}\right|+\frac{a^{2}}{4}
$$

On voit que le rayon croîtrait avec le temps, et le débit de fuite ne serait jamais nul.

Et pour chaque phase d'infiltration d'eau (c'est-à-dire $t$ donné), on sait calculer l'état élastique du terrain.

Mais que se passe-t-il réellement dans le terrain? 
Il est incontestable que la force de gravité a une influence sur l'écoulement examiné plus haut. Ainsi la zone imprégnée d'eau d'infiltration est en réalité une couche ovale, mais assimilable seulement pour $t$ petit (c'est-à-dire $b$ petil) à une couche cylindrique, comme l'indiquent lés figures ci-dessous :
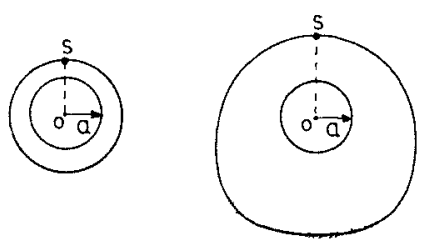

FIG. A

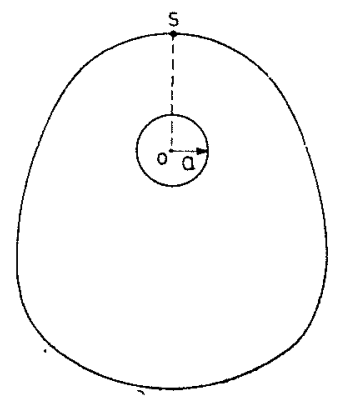

et celte couche ovale pourrait atteindre théoriquement un régime permanent d'allure lelle que le montre la figure suivante :

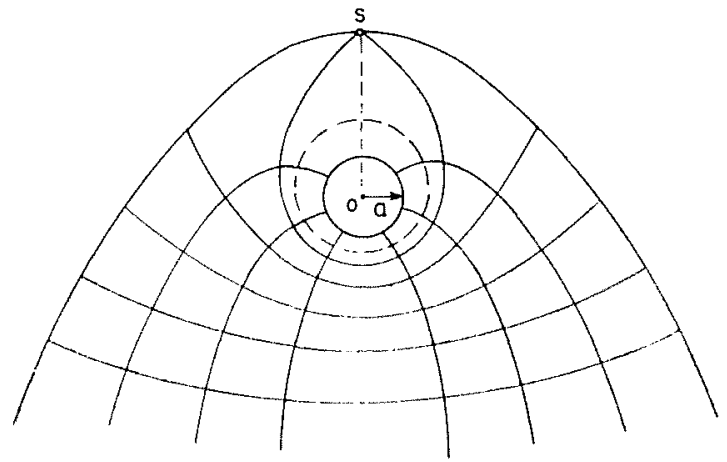

FiG. B

et le calcul de ce cas est possible mathématiquement.

Mais physiquement, il n'est pas concevable que l'eau puisse couler indéfiniment vers le fond du terrain. Ainsi cet écoulement d'infiltration finirait par rencontrer à distance finie une nappe qui ne lui permettrait plus de continuer à couler verticalement.

Or, une fois que l'auréole imprégnée d'eau d'infiltration atteint le niveau imperméable, supposé ici horizontal, l'écoulement d'infiltration évolue et se présentera avec l'allure suivante :

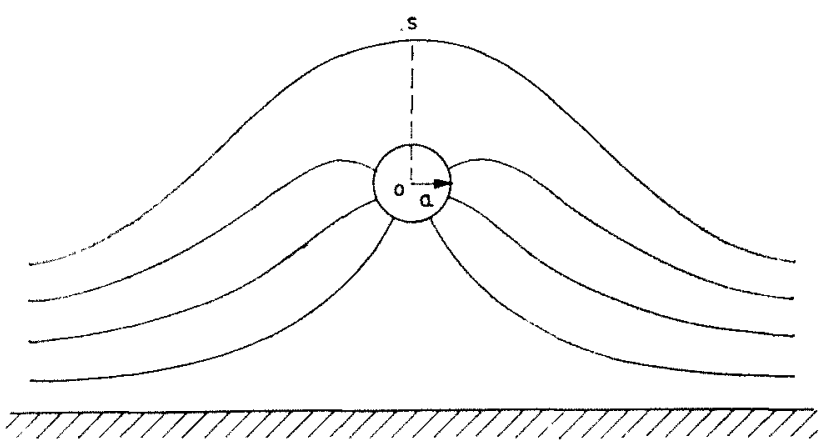

FiG. C

Que se passe-t-il ensuite?

Il est logique que la position de la surface libre du terrain joue à son tour un rôle déterminant. En effet, si la surface libre était très éloignée vers les deux côtés, la courbe qui limite la zone impré- 
gnée d'eau d'infiltration lendrait théoriquement vers une horizontale à la distance $\mathrm{H}_{0}$ au-dessus de la galerie. Mais si la surface libre du terrain est très éloignée d'un côté, el à distance finie de l'autre, la courbe indiqué plus haut aurait une asymplote horizontale vers le côté terrain et l'allure parabolique vers l'autre côté.

Ces considérations montrent clairement que la recherche du régime permanent se heurte à de très grosses difficultés, car, de toutes façons, les hypothèses prises à la base de cette recherche joueront un rôle déterminant dans le résultat obtenu; et on n'a, a priori, que tròs peu de raisons d'adopter une certaine hypothèse au départ plutôt qu'une autre. Ainsi, le problème concret est très complexe, et dépend étroitement des conditions locales, difficiles à déterminer.

Comme les calculs précis sont très difficiles et aléatoires par rapport à la réalité, nous pensons que le raisonnement suivant, fournissant des résultats simples, pourrait être très utile dans la pratique industrielle.

On sait qu'à chaque phase de l'infiltration, il existe autour de l'ouverture circulaire une région où $d \mathrm{H} / d n$ est assez grand. Il est à remarqer que la grandeur de cette région, et la distance $d=$ OS (voir les figures plus haut), dépendent évidemment de $\mathrm{H}_{0}\left(d<\mathrm{H}_{0}\right)$.

Appliquons alors la loi de Darcy de l'écoulement radial dans la région que nous venons d'indiquer. On voit que $Q$, le débit total de fuite par unité de longueur de la galerie, satisfait à la relation :

$$
\mathrm{Q}<\frac{2 \pi k \mathrm{H}_{0}}{\log (d / a)}
$$

En efret, l'écoulement réel à cet instant ne pourrait s'assimiler à celui d'un exutoire sous un rayon égal à $d$, que sur une portion de la circonférence totale.

Ón remarque là une approximation analogue — au sens de l'écoulement près - à celle provenant de l'interprétation du pompage dans une nappe, avec intervention du « rayon d'action » qui a déjà soulevé tant de controverses.

Et dans la pratique, on peut, à notre avis, prendre pour $t$ grand l'expression :

$$
\mathrm{Q}=\frac{2 \pi k \mathrm{H}_{0}}{\log \left(\mathrm{H}_{0} / \alpha\right)}
$$

pour avoir l'ordre de grandeur de $Q$ dans la réalité. Par exemple, pour une galerie en charge sous 100 mètres d'eau en bon rocher, de perméabilité $k=10^{-6} \mathrm{~m} / \mathrm{s}$, de rayon $a=2$ mètres, on trouve un débit $Q$ égal à $0,16 \mathrm{l} / \mathrm{s}$ par mètre de galerie. Ce chiffre est en général supérieur à la valeur réelle.

Il est très intéressant d'évaluer d'autre part le temps T nécessaire pour que l'auréole imprégnée d'eau puisse être considérée comme permanente (c'est-à-dire $d$ assez grand). La façon la plus simple, pour avoir l'ordre de grandeur de $\mathrm{T}$, e'est de remplacer dans (31) $b$ par $\mathbf{H}_{0}$. Ainsi, on trouve tris facilement pour le cas indiqué ci-dessus, et en prenant $m=0,05$, que l'ordre de grandeur de T est de $p l u-$ sieurs annes (toujours avec $k=10^{-6} \mathrm{~m} / \mathrm{s}$ ). Nous remarquerons que ce temps est directement proportionnel au coefficient de porosité et inversement proportionmel au coelficient de perméabilité. Mais nous n'en retiendrons ici que l'ordre de grandeur, qui est considérable et qui nous amène aux conclusions suivantes, compte tenu de résultats du calcul élastique indiqué dans la première partie:

- Si une pression est appliquée de façon permanente dans une galerie au rocher, les contraintes du terrain dues à la mise en pression du terrain sont extrêmement faibles : le terme $d \mathrm{P} / d r$ de l'équation (9) de la première partie est très réduit.

- Le terrain se mel en équilibre de pression avec l'intérieur de la galerie, mais cette mise en équilibre demande un lemps considérable et représente des volumes d'eau importants.

Il paraît intéressant d'examiner ce qui se passe lorsque les pressions appliquées varient.

\section{PRESSIONS VARIABLES}

La plupart des aménagements hydroélectriques modernes sont équipés de galeries en charge qui alimentent directement l'usine de production d'énergie. Or, le débit pris par l'usine doit suivre exactement la demande d'énergie, d'où des variations soudaines de débit. Celles-ci sont absorbées ou fournies par la cheminée d'équilibre, au prix de variations de la pression dans la galerie d'amenée, dont la période est de l'ordre de grandeur de celle du système oscillant galerie-cheminée, donc de quelques 
minutes. Cette durée peut être considérée comme nulle devant le temps de mise en pression du lerrain, de sorte que la distribution de la pression hydraulique dans le terrain le long d'un rayon serait de la forme :

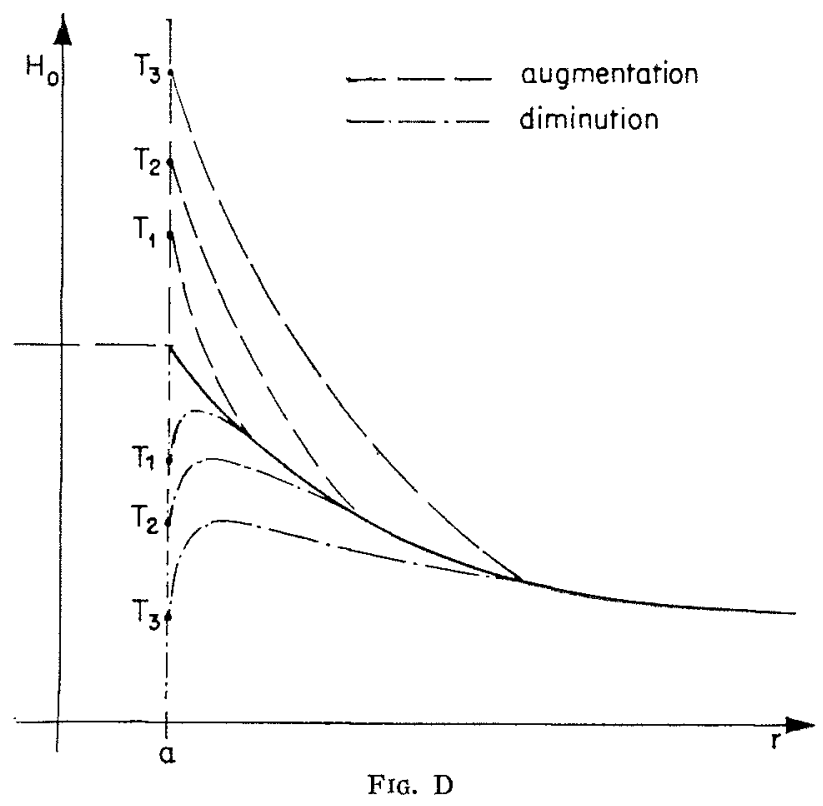

c'est-à-dire qu'à l'intérieur du terrain, la pression interstitielle reste presque inchangée et l'effet de la variation de $\mathrm{H}_{0}$ se comporte comme si e'était un simple effet de surface. Done, du point de vue de l'état élastique du terrain, les calculs se font en ajoutant à l'état du régime un état supplémentaire dù à la variation de pression appliquée seulement à la surface intérieure de la galerie.

Mais à une variation de pression $\mathrm{H}_{0}$ dans la galerie, sera lié un débit positif ou négatif du tcrrain vers la galerie. L'ordre de grandeur des variations de pression peut être assez élevé et atteindre dans certains cas facilement 30 à 40 mètres : la cheminée d'équilibre est souvent constituée par un puits de faible section entre le niveau maximum et le niveau minimum, de sorte que, pour une variation de débit même faible, l'oscillation peut être importante.

S'il s'agit d'une surpression, le débit de fuite serait simplement augmenté, mais pendant un temps très court, ce qui est parfaitement négligeable si on considère le temps de mise en pression du terrain. Et il n'y a pratiquement pas de risque.

Par contre, s'il s'agit d'une dépression, l'écoulement aura lieu du terrain vers la galerie. Théoriquement, le calcul que nous avons présenté admet que le débit est parfaitement réparti entre les fissures du rocher. Pratiquement, il en va certainement autrement, et même si on peut définir un coefficient de perméabilité « moyen », la circulation de l'eau s'effectue essentiellement par des diaclases du terrain, quelquefois remplies de roches décomposées. Ces fragments de roche risquent d'être entrainés vers la galerie, en cas de diminution de la pression, si la galerie n'est pas revêtue. Ceci ne présente peut-être pas tellement d'inconvénients si la couverture est grande: les fissures risquent peu de déboucher en surface. Mais le risque apparaît si la couverture est assez réduite.

Ces considérations nous aminent aux conclusions suivantes:

$1^{\circ}$ Il est dangereux de prévoir un système de cheminée d'équilibre qui entrainerait dans la galerie des variations de pression trop fortes.

Par contre, la durée d'apparition de ces variations de pression importe peu : elle est pratiquement toujours nulle devant la constante de temps des autres phénomènes.

$2^{\circ}$ Le danger dépend de la qualité du rocher. Si celni-ci n'est pas revêtu et renferme des zones diaclasées avec remplissage de roche décomposée, il peut $\mathrm{y}$ avoir débourrage de fissures; ceci est dangereux au cas où la couverture de terrain ne serait pas suffisante.

Que se passe-t-il si la galerie est revêtue?

Si la galerie est revêtue en héton ou en gunite de coefficient de perméabilité $k_{1}$ et de porosité $m_{1}$ jusqu'à la distance $a_{1}$, on sait que les formules (29), (30) et (31) restent valables pour le début de la mise en eau de la galerie, à condition d'y remplacer respectivement $k, m$ par $k_{1}, m_{1}$. 
Notons en passant que l'imprégnation du revêtement seul dure normalement assez peu de temps : c'est de l'ordre de quelques heures en général. En effet, pour imprégner un revêtement en gunite de $k_{1}=10^{-8} \mathrm{~m} / \mathrm{s}$, d'une épaisseur de $4 \mathrm{~cm}$, pour une galerie de $a=2 \mathrm{~m}$ sous la charge de $\mathrm{H}_{0}=100 \mathrm{~m}$, le temps est de l'ordre de :

$$
110 \times m_{1} \text { heures }
$$

Mais ce temps reste relativement très grand par rapport à la période des oscillations hydrauliques dans la galerie.

Une fois que la zone imprégnée d'eau dépasse le revêtement et comprend à son intérieur une couche de terrain, on a, si $\mathrm{H}_{1}$ est la pression hydraulique entre le revêtement et le terrain :

$$
\mathrm{H}_{1}=\mathrm{H}_{0}-\frac{q}{k_{1}} \log \frac{a_{1}}{a}
$$

pour le revêtement, et : ,

$$
\mathrm{H}_{(t)}=\mathrm{H}_{1}-\frac{q}{k} \log \frac{r}{a_{1}} \quad\left(b>r>a_{1}\right)
$$

à l'intérieur du terrain. D'où :

$$
\frac{\mathrm{H}_{0}-\mathrm{H}}{q}=\frac{1}{k}\left[\log \frac{r}{a_{1}}+\frac{k}{k_{1}} \log \frac{a_{1}}{a}\right]
$$

Le deuxième terme du second membre représente la perte de charge dans le revêtement; et le premier, la répartition de la pression hydraulique dans le terrain. Ceci est valable pour $b$ voisin de $a_{1}$. Ecrivons alor's que $a_{1}=a+\varepsilon, \varepsilon / a$ étant en général très petit. Par exemple, $\varepsilon / a$ est de l'ordre de 0,10 , s'il s'agit d'un revêtement en béton, et de l'ordre de 0,01 s'il s'agit d'un revètement en gunite ou en béton projeté; on voit que :

$$
\log \left(1+\frac{\bar{\varepsilon}}{a}\right) \# \frac{\bar{\varepsilon}}{a}
$$

(32) devient finalement :

$$
\frac{\mathrm{H}_{0}-\mathrm{H}}{q}+\frac{1}{k}\left[\log \frac{\underline{r}^{\prime}}{a_{1}}+\frac{k}{k_{1}} \frac{\dot{e}}{a}\right]
$$

Dans la pratique, le revêtement est nettement plus imperméable que le rocher, c'est-à-dire que $k / k_{1}$ est grand; on en déduit que le revêtement supporte une part importante de la totalité de la pression $\mathrm{H}_{0}$.

Lorsque la zone du rocher imprégnée d'eau d'infiltation devient plus grande, les relations trouvées ci-dessus seront moins exactes; et on aura dans le rocher des écoulements semblables à ceux que nous avons indiqués à propos des galeries non revêtues. La seule différence entre les deux phénomènes est qu'ici la pression interstitielle appliquée sur le rocher est $\mathrm{H}_{1}$, (qui représente une fraction de $\mathrm{H}_{0}$, variable suivant la grandeur de la zone imprégnée). Dans les calculs précis, il faut remplacer dans (32) le premier terme du second membre par un autre terme déduit des lois d'écoulement plus adéquates; l'application directe de (32) dans ce cas donnera des valeurs approchées.

Pour avoir un ordre de grandeur de la répartition des pressions lorsque la zone imprégnée dans le rocher est très grande, on peut pratiquement utiliser (32) en y remplaçant $r$ par $d$ (qui est assez grand) avec $d<\mathrm{H}_{1}$. Ainsi, on voit que pour le revêtement de gunite d'épaisseur 4 cm dans une galerie de $a=2$ mètres, sous la charge $H_{0}=100 \mathrm{~m}$,

$$
\frac{r}{a_{1}}<50
$$

si $k_{1}=10^{-\mathrm{s}} \mathrm{m} / \mathrm{s}, k=10^{-6} \mathrm{~m} / \mathrm{s}, k / k_{1}=100$, la répartition de la pression hydraulique d'après (32) sera dans la proportion de 2 pour le revêtement et 3,9 pour le rocher. C'est-à-dire que le revêtement prendrait le tiers et le rocher, le reste.

Ainsi, au début de la mise en eau de la galerie, le revêtement prend la totalité de la pression $\mathrm{H}_{0}$, et au fur et à mesure que l'eau s'infiltre dans le rocher, la charge appliquée sur le revêtement sera réduite jusqu'à une portion de la pression totale $\mathrm{H}_{0}$. Pour l'exemple indiqué, cette portion est d'ordre $1 / 3$ environ. Mais il est à remarquer que si $k / k_{1}$ était plus grand, cette portion serait plus grande. 
Examinons maintenant ce qui se passe pour une variation de pression dans la galerie.

Comme la période d'oscillations de la pression $\mathrm{H}_{0}$ dans la galerie est encore trop petite par rapport à la durée de l'imprégnation du revêtement, on peut (pour la même raison que celle que nous avons indiquée dans le cas d'une galerie non revêtue) considérer que cette variation n'agit, au point de vue de l'état élastique de la galerie, que superficiellement, à la surface intérieure du revêtement. Ainsi, pendant l'augmentation de la pression $\vec{H}_{0}$, le revêtement subit une traction circulaire plus forte et s'appuie davantage sur le rocher; ceci n'a pas beaucoup d'inconvénients. Par contre, pour une diminution de $\mathrm{H}_{0}$, le revêtement a tendance à se décoller du rocher, et cette tendance provoquerail des écoulements du rocher vers le revêtement. Or, ils s'effectuent, comme nous l'avons dit plus haut, par des diaclases du rocher. Des détériorations locales du revêtement sont à craindre, surtout lorsque ce dernier est d'épaisseur très faible.

Ceci nous paraît constituer une raison suffisante pour ne pas employer de revêtements trop imperméables, par comparaison à leur résistance aux sous-pressions, dans une galerie en charge, surtout si la pression risque de varier beaucoup - et d'employer, quand les oscillations de pression risquent d'être élevées, une section revêtue en béton, de forme circulaire, pour résister aux pressions provenant de l'effet que nous venons de signaler.

\section{CONCLUSION}

Nous avons indiqué dans cette étude les éléments nécessaires pour étudier les galeries en charge, les formules utiles pour calculer les états élastiques et les considérations hydrauliques indispensables qui sont des données de base. En effet, le problème de résistance de galeries en charge aux pressions intérieures n'est pas seulement un probl’me de résistance mécanique des matériaux en présence de revêtement et de rocher, mais il s'agit surtout d'un problème d'écoulement où les perméabilités respectives jouent un rôle important.

D'après les quelques considérations exposées très rapidement dans la deuxième partie, la galerie avec un revêtement de faible épaisseur présente des risques. Et ces risques sont d'autant plus grands que le revêtement est mieux fait.

Ceci conduit à recommander, pour une galerie en charge, soit l'adoption d'un profil entièrement brut, mais seulement si le rocher est vraiment excellent (en particulier s'il n'existe pas de fissures bourrées de roches décomposées), soit l'adoption d'un profil revêtu, de forme intérieure circulaire, de façon à résister à des sous-pressions notables en cas de diminution de la pression intérieure en régime transitoire.

Bien entendu, les considérations développées ei-dessus, concernant un écoulement cylindrique, pourraient être appliquées au cas d'un écoulement quelconque dans le rocher, survenant à la suite de la mise en eau d'un barrage, par exemple. On conçoit en particulier combien les efforts appliqués et surtout le mode de résistance du terrain peuvent varier considérablement, suivant la répartition des perméabilités naturelles. D'où l'intérêt de «fixer» ces perméabilités - ne serait-ce que pour les mieux connaitre -, soit à l'aide d'un rideau d'injections si le terrain le permet, soit à l'aide d'un drainage du massif si le terrain est trop peu fissuré pour être injecté. 